\title{
Integrating mental and physical health assessment in a neuro-otology clinic: feasibility, acceptability, associations and prevalence of common mental health disorders
}

\author{
Authors: David Herdman, ${ }^{\mathrm{A}}$ Helen Sharma, ${ }^{\mathrm{B}}$ Anna Simpson ${ }^{\mathrm{C}}$ and Louisa Murdin ${ }^{\mathrm{D}}$
}

People with persistent physical symptoms are at risk of psychological symptoms, although recognition in medical settings is low. This is a retrospective observational study of 954 patients in a hospital outpatient neuro-otology clinic in order to assess the feasibility and acceptability of an electronic informatics system for collection of patient-reported outcomes, with real-time feedback to guide clinical care and describe the prevalence of anxiety and depressive symptoms.

The proportion of patients successfully completing the screen was high (70\%). The decline rate was low (5\%). The most common reason to decline was lack of confidence with technology. The prevalence of probable depression was $21 \%$ and for probable anxiety was $29 \%$. Suicidal ideation was present in $5 \%$. Anxiety and depression were highly correlated to dizziness specific outcome measures $(p<0.01)$.

Electronic screening is feasible and acceptable to patients and staff in this setting, helping to identify service needs, inform care and monitor outcomes.

KEYWORDS: Anxiety, depression, dizziness, vertigo, neuro-otology

\section{Introduction}

Mental health comorbidities are present in around $30-50 \%$ of patients presenting to outpatient clinics with vertigo and dizziness symptoms. ${ }^{1,2}$ The most common comorbidities are depression and anxiety. ${ }^{2}$ The relationship between mental illness and chronic vertigo and dizziness syndromes is complex and bidirectional, although there is clear evidence that depression and anxiety contribute to poorer health outcomes. ${ }^{3}$ The research literature also indicates that when services address the mental and physical

Authors: A physiotherapist, Guy's \& St Thomas' NHS Foundation Trust, London, UK and research fellow, King's College London, London, UK; Bhead of practice, Chartered Society of Physiotherapy, London, UK; ' project coordinator, King's College London, London, UK; 'Daudiovestibular physician, Guy's \& St Thomas' NHS Foundation Trust, London, UK and honorary associate professor, UCL Ear Institute, London, UK impact of chronic vertigo and dizziness together, patient outcomes (disease activity, functioning, quality of life) usually improve. ${ }^{4}$ Anxiety and depression exist on a continuum of severity and the point at which psychological distress becomes mental disorder is not clear-cut. Yet clinicians and patients sometimes need to make black and white decisions, such as when to seek help from mental health services. Recognition of psychological disorders by non-mental health professionals is low compared with structured clinical interview even in highly specialised neuro-otology clinics, meaning that many patients do not receive psychological interventions that are known to be effective. ${ }^{5,6}$ Even when problems are identified, healthcare teams are often illequipped to provide effective care due to limited access to expert psychological support. ${ }^{7}$ A structured screening procedure could be a valid alternative since it is not realistic to have psychiatrists or psychologists perform detailed standardised interviews in a routine setting.

Screening and assessment tools can help some patients, who may not otherwise relate their symptoms to mental ill health where appropriate, or who may feel unable to raise concerns about their thoughts and mood with their healthcare professional. These tools can also help healthcare professionals who would otherwise be reticent to probe patients' psychological problems and improve their ability to identify patients who may benefit from psychological support. Since completion of an assessment tool is often an important part of triage to talking therapies, early assessment could lead to more effective signposting and onward referral which would enable the patient to access the right care earlier in their pathway.

This paper is the first to describe the implementation of Integrating Mental \& Physical healthcare: Research Training and Services (IMPARTS) service development platform in a multidisciplinary neuro-otology clinic. ${ }^{8}$ IMPARTS is an integrated care package that supports clinical services in detecting and managing depression and anxiety among patients attending hospital for physical health conditions. Using paper questionnaires is time consuming to complete and analyse, and the results alone do not clearly guide management. IMPARTS has developed an electronic informatics system that facilitates routine collection of patient-reported outcomes, with real-time feedback, uploaded to the electronic patient record (EPR) to guide clinical care according to a structured protocol. The system creates a database that can be used for audit and research and has been implemented 
in medical specialties such as rheumatology, dermatology and chronic pain. ${ }^{9}$

Screening for anxiety and depression in patients with vertigo and dizziness can be challenging since they are often concerned about their symptoms being labelled or judged as purely psychological or even imaginary. Many patients with vertigo and dizziness disorders also report symptoms related to visual motion and so find using computer screens difficult. There was therefore also a concern that this method of screening may be problematic for this particular patient group. We therefore aim to describe the feasibility and acceptability of universal digital mental health screening in a neuro-otology clinic; validated physical outcome measures including the level of dizziness related handicap, disability, visual vertigo, headache, anxiety and depression including suicidal ideation identified; and the cross-sectional relationships between the physical and mental outcome measures.

\section{Methods}

\section{Study design}

This is a retrospective data analysis of the results from the first year of implementing an informatics screening tool in the tertiary multidisciplinary balance clinic at Guy's Hospital, London, from January 2017 to January 2018.

\section{Ethical considerations}

IMPARTS has generic ethical approval for a pseudonymised research database (REC reference: 18/SC/0039), with additional governance of approval from the IMPARTS patient-led research oversight committee. Institutional approval was granted from Guy's and St Thomas' NHS Foundation Trust.

\section{Consent}

No patient information or patient identifiable images are included in this article.

\section{Procedure}

Consecutive patients who attended the tertiary multidisciplinary balance clinic were invited to complete questionnaires on a touch-screen e-tablet by the receptionists while waiting for their appointment. After logging on, using their hospital identification number, patients completed a series of outcome measures concerning their mental and physical health. Some physical outcome measures were adapted using branching structures so that patients did not have to complete all measures if they were not relevant. The responses transferred in real-time to the EPR where a message also alerted the clinician if a patient had screened above the relevant predetermined threshold and provided advice on action to take, based on the severity of the patient's symptoms.

\section{Measures}

Outcome measures were selected in consultation with the clinical team to capture data pertinent to this patient group. To make the questionnaires more acceptable to patients, they were ordered so that the psychological measures appeared at the end. It took patients between 10-20 minutes to complete.
All patients completed the Dizziness Handicap Inventory, which is a validated self-report of dizziness related interference. ${ }^{10}$ Patients were then asked whether they experienced dizziness in visual contexts (eg supermarkets or watching moving objects). If they answered 'yes', the Situational Characteristics Questionnaire was presented to measure symptoms of visually induced dizziness. ${ }^{11}$ The next branching question asked about the presence of headaches and those patients who experienced headaches completed the Headache Impact Test- 6 and scores $\geq 50$ were flagged up on the EPR as 'high-impact headache' with instructions for the clinician to 'check for headache plan and consider analgesic overuse.'. The level of disability due to dizziness was assessed using the disability rating scale in which the patient was asked to select their level of disability based on six statements ranging from 'negligible symptoms' to 'unable to work for more than 1 year or established permanent disability.'13

Depression was assessed using the Patient Health Questionnaire-9 (PHQ-9) and anxiety was assessed using the Generalised Anxiety Disorders-7 Questionnaire (GAD-7). ${ }^{14,15}$ These questionnaires have been widely validated in physically ill populations and are the current tools used by Improving Access to Psychological Therapy (IAPT) services in the UK to determine suitability for talking therapies. For depression status there were three options.

> Probable major depression. Either item 1 or 2 of the PHQ-9 was 'more than half the days' or above and five or more of the nine PHQ-9 items were at least 'more than half the days' except for item nine (suicidal ideation) which only counted towards the diagnosis if checked at least 'several days'.

> Some depressive symptoms. If either 1 or 2 of the PHQ-9 were 'more than half the days' or above.

> Depression screen negative.

An alert also appeared on the patient record to indicate suicidal ideation if the patient responded 'more than half the days' or 'nearly every day' to item nine ('have you been bothered by thoughts that you would be better off dead or of hurting yourself in some way'). Criteria for probable generalised anxiety disorder was met if patients scored $\geq 10$ on the GAD-7. Scores of 5-9 indicated some symptoms of anxiety.

\section{Care pathways}

All clinical staff undertook three 1-hour training sessions in core mental health skills so that they would feel confident discussing and managing psychological distress. If patients screened positive for probable major depression, this was discussed with them during their consultation and advice on referral was displayed according to the severity (Fig 1). A risk assessment was undertaken for patients who screened positive for suicidal ideation and if there were immediate concerns about the patient's safety, the duty liaison psychiatrist was contacted.

If patients screened positive for mild/moderate depression, or probable generalised anxiety, their general practitioner was informed in the clinical report and patients were informed about self-referral to IAPT services. IAPT is run by the NHS and provides evidence-based mental health support and treatment for people with mild to moderate depression and anxiety. If patients screened positive for more than one problem, then the psychiatry referral would take precedent. The referral information was for guidance only and referral was only offered after discussion with the patient. 


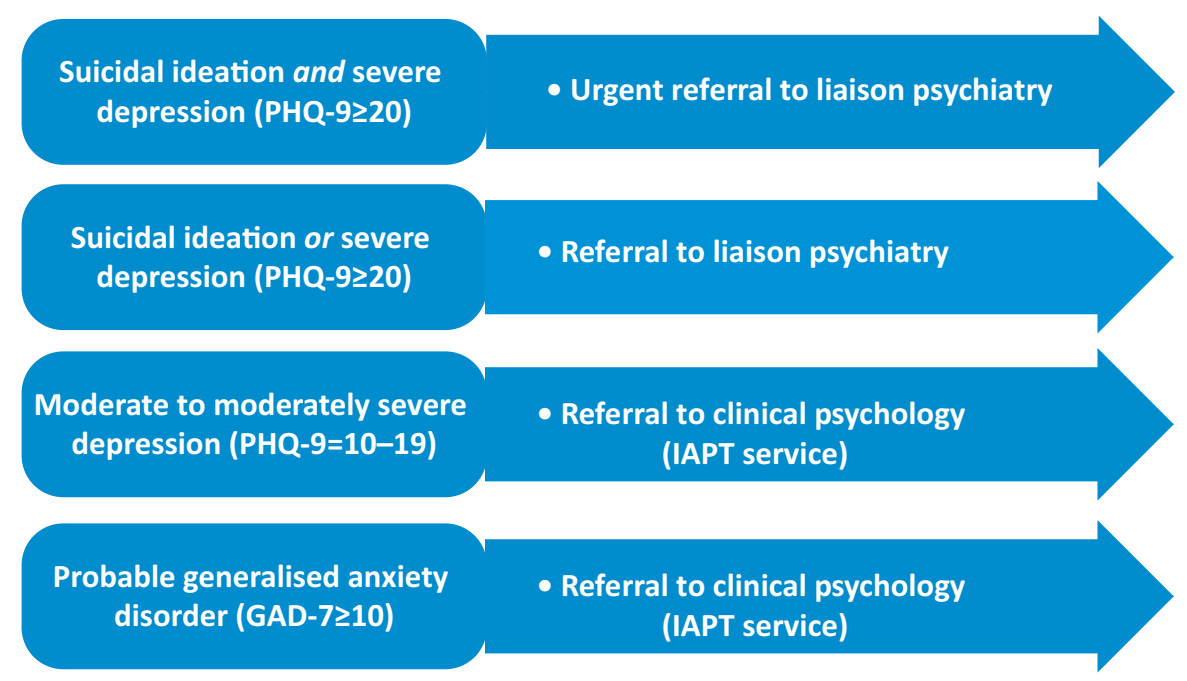

Fig 1. Referral guidelines for patients who screened positive for depression and anxiety. IAPT = Improving Access to Psychological Therapies; GAD-7 $=$ Generalised Anxiety Disorder-7 Questionnaire; PHQ-9 = Patient Health Questionnaire-9.

\section{Data analysis}

Feasibility was quantified as the proportion of patients attending their appointment who completed the IMPARTS questionnaire. Acceptability was quantified as the inverse of the proportion of patients declining screening. Group differences were analysed using a two-sample t-test and $\chi^{2}$ test. If patients did not complete screening, the assessing clinicians manually recorded the reason for non-completion using a separate form.

The prevalence of probable depression and anxiety was expressed as the percentage of cases determined by the PHQ-9 and GAD-7. Means and standard deviations were also calculated for the other baseline screening responses and intercorrelations were determined using a partial Pearson's $r$ coefficient controlling for age and gender.

\section{Results}

\section{Participants}

Nine-hundred and fifty-four (78\%) patients completed screening at their first visit. Table 1 shows the age and gender comparison for patients who did and did not complete screening. A higher proportion of females declined screening but the difference between males and females was not significant ( $p=0.057)$. However, patients who did not complete screening were significantly older $(p<0.01)$.

\section{Feasibility and acceptability}

Only $5 \%$ of the total proportion of patients declined screening, which was mainly due to lack of confidence with IT (Table 2). Only $3 \%$ of the non-responders declined due to dizziness symptoms.
The top reason for not completing screening was reception staff not providing the e-tablet to the patient on arrival, followed by technical errors with the internet connection of the tablet.

\section{Prevalence}

Table 3 shows the percentages of patients who screened above the thresholds for depression and anxiety and referral recommendations. Numerators are different as they reflect the duration in which the relevant measures appeared on the system. Thirty-two per cent of patients had either anxiety or depression, and $18 \%$ had both. Prevalence for anxiety $(29 \%)$ was slightly higher than probable major depression $(21 \%)$ and $10 \%$ required referral to liaison psychiatry. Five per cent of patients reported suicidal ideation. Table 4 shows the percentages of categories for the physical outcome measures.

\section{Correlations}

Anxiety and depressive symptoms were significantly associated with the self-reported outcomes even after controlling for age and gender (see Table 5). There were also significant intercorrelations between all other measures (all correlations $\mathrm{p}<0.01$ ).

\section{Discussion}

\section{Synopsis of key findings}

This study found that using an informatics system to screen for common mental health problems in a neuro-otology clinic was feasible and acceptable to patients. Older adults were less likely to complete screening, possibly due to lower confidence with an electronic device. The main reason for not completing the

\section{Table 1. Demographic characteristics and differences between completers and non-completers}

$\begin{array}{llll} & \text { Completers }(\mathrm{n}=\mathbf{9 5 4}) & \text { Non-completers }(\mathrm{n}=\mathbf{2 7 0}) & \\ \text { Age, years, mean } \pm \text { SD (range) } & 51.48 \pm 16.0(16-90) & 59.61 \pm 16.15(18-96) & t(1,222)=-7.32, p<0.01 \\ \text { Gender, female, \% }(n) & 70(664) & 76(204) & \chi^{2}(1, n=1,224)=3.62, p=0.057\end{array}$


Table 2. Proportion of patients screened, and

reasons that they were not screened

\begin{tabular}{|c|c|}
\hline & n (\%) \\
\hline Attended appointment & $1,224(100)$ \\
\hline Completed screening & $954(78)$ \\
\hline \multicolumn{2}{|l|}{ Reasons for declining: } \\
\hline Confidence with technology & $33(2.7)$ \\
\hline Due to dizziness & $8(0.7)$ \\
\hline Similar questionnaires already completed & $6(0.5)$ \\
\hline $\begin{array}{l}\text { Declined other (eg they wanted to watch } \\
\text { television in the waiting area or no reason } \\
\text { given) }\end{array}$ & $13(1.1)$ \\
\hline \multicolumn{2}{|l|}{ Other reasons not screened: } \\
\hline E-tablet not provided or available & $113(9.2)$ \\
\hline Network error / technical problem & $26(2.1)$ \\
\hline Insufficient English & $25(2.0)$ \\
\hline Arrived late / not enough time & $22(1.8)$ \\
\hline Other $^{a}$ & $24(2.0)$ \\
\hline
\end{tabular}

questionnaires was reception staff forgetting to hand them to the patient. Only $5 \%$ of patients didn't want to complete the questionnaires and, perhaps surprisingly, visually induced dizziness was not a significant barrier to using the e-tablet.

Anxiety and depressive symptoms were common and over 30\% of patients fulfilled criteria for onward referral. This is consistent with literature from other neuro-otology clinics although the prevalence of depressive symptoms was a little higher in our sample. ${ }^{16}$ Detecting emotional disorders in patients with vertigo and dizziness is important, not only because it is common, but because these factors have been shown to adversely affect the treatment outcomes and recurrence of vestibular diseases. ${ }^{17-19}$

This is the first study to our knowledge that has reported prevalence of suicidal thoughts in this setting. We found $5 \%$ of patients had suicidal ideation. There is a widespread fear that asking about suicide may make individuals more likely to engage in suicidal thoughts or behaviours, although there is now strong evidence to refute this. ${ }^{20}$ Notably, less than a third of suicide completers have had contact with mental health services and a much larger proportion seek other healthcare contact. ${ }^{21}$ Healthcare professionals working with patients with chronic vertigo and dizziness disorders should therefore be alert to suicidal risks and trained in assessment and appropriate support of these.

Anxiety and depressive symptoms were also highly correlated with handicap, visually induced dizziness, headache severity and disability. Levels of somatic complaints are highly prevalent in this population and more than half of patients reported a high impact headache, supporting the emerging literature that suggests it is also important to consider comorbid headache in patients with chronic dizziness. ${ }^{22}$

Little is known about the occupational impact of vertigo and dizziness. More than $10 \%$ of our patients reported work related disability, with $5.8 \%$ of those on established sick leave for more than 1 year. Although these numbers are less than previously reported, many patients still experienced disruptions to their usual activities. ${ }^{23}$
Table 3. Prevalence of mental health status and referral guidelines according to PHQ-9 and GAD-7

\begin{tabular}{|c|c|c|}
\hline & Category & n (\%) \\
\hline \multirow[t]{3}{*}{ Depression (diagnosis) } & No symptoms & $437(70.0)$ \\
\hline & Some symptoms & $54(8.7)$ \\
\hline & Probable major depression & $133(21.3)$ \\
\hline \multirow[t]{3}{*}{ Referral (PMD) } & Alert GP in letter; IAPT referral & $71(11.4)$ \\
\hline & Referral to liaison psychiatry & $43(6.9)$ \\
\hline & $\begin{array}{l}\text { Urgent referral to liaison } \\
\text { psychiatry }\end{array}$ & $19(3.0)$ \\
\hline \multirow[t]{3}{*}{ Suicidal ideation } & Suicidal ideation & $34(5.4)$ \\
\hline & $\begin{array}{l}\text { Suicidal ideation or severe } \\
\text { depression }\end{array}$ & $51(8.2)$ \\
\hline & $\begin{array}{l}\text { Suicidal ideation and severe } \\
\text { depression }\end{array}$ & $19(3.0)$ \\
\hline \multirow[t]{5}{*}{ Depression (severity) } & None & $234(37.5)$ \\
\hline & Mild & $186(29.8)$ \\
\hline & Moderate & $81(13.0)$ \\
\hline & Moderately severe & $68(10.9)$ \\
\hline & Severe & $55(8.8)$ \\
\hline \multirow[t]{3}{*}{ Anxiety } & No symptoms & $417(46)$ \\
\hline & Mild symptoms & $228(25.2)$ \\
\hline & $\begin{array}{l}\text { Probable generalised anxiety } \\
\text { (IAPT referral) }\end{array}$ & $261(28.8)$ \\
\hline \multirow[t]{2}{*}{ Anxiety or depression } & No & $417(67.7)$ \\
\hline & Yes & $199(32.3)$ \\
\hline \multirow[t]{2}{*}{ Anxiety and depression } & No & $504(81.8)$ \\
\hline & Yes & $112(18.2)$ \\
\hline
\end{tabular}

GAD-7 = Generalised Anxiety Disorder-7 Questionnaire; GP = general practitioner; IAPT = Improving Access to Psychological Therapies service; PMD = probable major depression; PHQ-9 $=$ Patient Health Questionnaire-9.

\section{Strengths of the study}

This is the first study we are aware of to report the feasibility and acceptability of electronic physical and mental health screening in a tertiary neuro-otology clinic. The findings can also be used in other physical healthcare settings to consider factors that may increase the proportion of patients completing screening. This study included a large sample of patients over 1 year and is therefore highly representative of the clinic.

\section{Limitations}

The data obtained on the reasons why patients did not complete screening relied on manual reporting by the physiotherapists involved in the clinic which might be prone to error. However, due to the diligence of the therapists and meticulous cross checking we believe the number of missing entries to be negligible.

Another limitation of the data is that it might overestimate referral rates, since we were not able to exclude individuals with pre-existing psychiatric illnesses or other comorbid conditions 
Table 4. Condition specific outcome measures

\begin{tabular}{lll} 
& Category & Percentage \\
DHI & Mild handicap (0-30) & $32.1 \%$ \\
& Moderate handicap (31-60) & $38.3 \%$ \\
& Severe handicap $(>60)$ & $29.7 \%$ \\
SCQ & Visual vertigo $(>0.7)$ & $52.4 \%$ \\
HIT-6 & High impact headache ( $\geq 50)$ & $54.0 \%$ \\
DRS & Negligible symptoms & $10.8 \%$ \\
& Bothersome symptoms & $28.3 \%$ \\
& Symptoms interfere with outside & $13.2 \%$ \\
& activities & \\
& Symptoms interfere with both usual & $37.0 \%$ \\
& work activities and outside activities & \\
& Unable to work or changed jobs & $4.9 \%$ \\
& Unable to work for more than 1 year or & $5.8 \%$ \\
& established permanent disability & \\
\hline
\end{tabular}

DHI = Dizziness Handicap Inventory; DRS = Disability Rating Scale; HIT-6 = Headache Impact Test-6; SCQ = Situational Characteristics Questionnaire.

(eg tinnitus). Furthermore, we were not able to report any association with diagnosis or clinical findings although we hope that this will be included in the next iteration of the system.

Additionally, it is not clear to what extent the screening protocol accurately identified patients who were suitable for mental health services. For example, the PHQ-9 item 9 often overestimates suicidal ideation. ${ }^{24}$ This is an important clinical consideration given the already considerable burden on mental health services. At all times, it was made clear that the guidance did not supersede clinical judgement but since screening naturally resulted in higher rates of referrals, liaison psychiatry services struggled to meet the demand, and this would be worth exploring further in the future.

Table 5. Summary of partial intercorrelation (Pearson's $r$ ), mean and standard deviation for scores on the self-reported measures after controlling for age and gender

\begin{tabular}{lllllll} 
& $\mathbf{1}$ & $\mathbf{2}$ & $\mathbf{3}$ & $\mathbf{4}$ & $\mathbf{5}$ & $\mathbf{6}$ \\
1. PHQ-9 & $\mathrm{n} / \mathrm{a}$ & $0.786^{* *}$ & $0.712^{* *}$ & $0.495^{* *}$ & $0.581^{* *}$ & $0.424^{* *}$ \\
2. GAD-7 & $\mathrm{nr}$ & $\mathrm{n} / \mathrm{a}$ & $0.611^{* *}$ & $0.490^{* *}$ & $0.489^{* *}$ & $0.370^{* *}$ \\
3. DHI & $\mathrm{nr}$ & $\mathrm{nr}$ & $\mathrm{n} / \mathrm{a}$ & $0.623^{* *}$ & $0.582^{* *}$ & $0.566^{* *}$ \\
4. SCQ & $\mathrm{nr}$ & $\mathrm{nr}$ & $\mathrm{nr}$ & $\mathrm{n} / \mathrm{a}$ & $0.376^{* *}$ & $0.407^{* *}$ \\
5. HIT-6 & $\mathrm{nr}$ & $\mathrm{nr}$ & $\mathrm{nr}$ & $\mathrm{nr}$ & $\mathrm{n} / \mathrm{a}$ & $0.420^{* *}$ \\
6. DRS & $\mathrm{nr}$ & $\mathrm{nr}$ & $\mathrm{nr}$ & $\mathrm{nr}$ & $\mathrm{nr}$ & $\mathrm{n} / \mathrm{a}$ \\
Mean & 8.06 & 6.64 & 45.78 & 1.05 & 59.05 & 2.16 \\
Standard & 6.84 & 6.19 & 24.77 & 1.10 & 9.07 & 1.32 \\
deviation & & & & & & \\
\hline
\end{tabular}

${ }^{* *}=p<0.01 ;$ DHI = Dizziness Handicap Inventory; DRS = Disability Rating Scale; GAD-7 = Generalised Anxiety Disorder-7 Questionnaire; HIT-6 = Headache Impact Test- $6 ; \mathrm{n} / \mathrm{a}=$ not applicable; $\mathrm{nr}=$ not required; $\mathrm{PHQ}-9=$ Patient Health Questionnaire-9; SCQ = Situational Characteristics Questionnaire.

\section{Clinical applicability of the study}

Electronic mental health screening tools can be administered by physicians, other doctors and allied healthcare professionals working in hospital outpatient or similar settings to help identify patients who may benefit from psychological support. Older adults may require further support to use electronic rather than paper questionnaires and services should seek to engage those staff responsible for approaching patients on a regular basis. To be useful, screening needs to be implemented alongside mental health training and there also needs to be a clear pathway to treatment and support for patients who screen positive.

\section{Conclusion}

Patients attending a specialist neuro-otology service are at risk of anxiety and depression, including suicidal thoughts, which are correlated with self-reported outcomes. Web-based mental health screening is feasible and acceptable although older adults may require more support. The presence of suicidal ideation reinforces the need for mental health assessment to be embedded in a locally agreed, robust pathway to maintain patient safety. Physical health services should consider ways to address the mental health needs of their patients to facilitate access to mental health services with the goal of improving treatment outcomes and decreasing associated social and economic burdens.

\section{References}

1 Persoons P, Luyckx K, Desloovere C, Vandenberghe J, Fischler B. Anxiety and mood disorders in otorhinolaryngology outpatients presenting with dizziness: validation of the self-administered PRIME-MD Patient Health Questionnaire and epidemiology. Gen Hosp Psychiatry 2003;25:316-23.

2 Lahmann C, Henningsen P, Brandt T et al. Psychiatric comorbidity and psychosocial impairment among patients with vertigo and diz ziness. J Neurol Neurosurg Psychiatry 2015;86:302-8.

3 Ketola S, Havia M, Appelberg B, Kentala E. Psychiatric symptoms in vertiginous patients. Nord J Psychiatry 2015;69:287-91.

4 Naber CM, Water-Schmeder O, Bohrer PS et al. Interdisciplinary treatment for vestibular dysfunction: the effectiveness of mindfulness, cognitive-behavioral techniques, and vestibular rehabilitation. Otolaryngol Head Neck Surg 2011;145:117-24.

5 Cepoiu M, Mccusker J, Cole MG et al. Recognition of depression by non-psychiatric physicians - A systematic literature review and meta-analysis. J Gen Intern Med 2008;23:25-36.

6 Limburg K, Dinkel A, Schmid-Mühlbauer G et al. Neurologists' assessment of mental comorbidity in patients with vertigo and dizziness in routine clinical care - comparison with a structured clinical interview. Front Neurol 2018;9:957.

7 Walker A, Kantaris X, Chambers M. Understanding therapeutic approaches to anxiety in vestibular rehabilitation: a qualitative study of specialist physiotherapists in the UK. Disabil Rehabil 2018;40:829-35.

8 Integrating Mental \& Physical healthcare: Research, Training \& Services. King's College London. www.kcl.ac.uk/ioppn/depts/pm/ research/imparts/index.aspx [Accessed 14 November2018].

9 Rayner L, Matcham F, Hutton J et al. Embedding integrated mental health assessment and management in general hospital settings: feasibility, acceptability and the prevalence of common mental disorder. Gen Hosp Psychiatry 2014;36:318-24.

10 Jacobson GP, Newman CW. The development of the Dizziness Handicap Inventory. Arch Otolaryngol Head Neck Surg 1990;116:424-7.

11 Guerraz M, Yardley L, Bertholon P et al. Visual vertigo: symptom assessment, spatial orientation and postural control. Brain 2001;124:1646-56. 
12 Kosinski M, Bayliss MS, Bjorner JB et al. A six-item short-form survey for measuring headache impact: the HIT-6. Qual Life Res 2003;12:963-74.

13 Shepard NT, Telian SA, Smith-Wheelock M. Habituation and balance retraining therapy. A retrospective review. Neurol Clin 1990;8:459-75.

14 Spitzer RL, Kroenke K, Williams JB. Validation and utility of a selfreport version of PRIME-MD: the PHQ primary care study. Primary Care Evaluation of Mental Disorders. Patient Health Questionnaire. JAMA 1999:282:1737-44.

15 Spitzer RL, Kroenke K, Williams JB, Lowe B. A brief measure for assessing generalized anxiety disorder: the GAD-7. Arch Intern Med 2006;166:1092-7.

16 Ketola S, Havia M, Appelberg B, Kentala E. Depressive symptoms underestimated in vertiginous patients. Otolaryngol Head Neck Surg 2007:137:312-5.

17 Wei W, Sayyid ZN, Ma X, Wang T, Dong Y. Presence of anxiety and depression symptoms affects the first time treatment efficacy and recurrence of benign paroxysmal positional vertigo. Front Neurol 2018;9:178.

18 Herdman SJ, Hall CD, Delaune W. Variables associated with outcome in patients with unilateral vestibular hypofunction. Neurorehabil Neural Repair 2012;26:151-62.

19 Yokota Y, Kitahara T, Sakagami M et al. Surgical results and psychological status in patients with intractable Meniere's disease. Auris Nasus Larynx 2016:43:287-91.
20 Blades CA, Stritzke WGK, Page AC, Brown JD. The benefits and risks of asking research participants about suicide: a meta-analysis of the impact of exposure to suicide-related content. Clin Psychol Rev 2018;64:1-12.

21 Luoma JB, Martin CE, Pearson JL. Contact with mental health and primary care providers before suicide: a review of the evidence. Am J Psychiatry 2002;159:909-16.

22 Goto F, Arai M, Arai M. The influence of headaches in patients with chronic dizziness. Acta Otolaryngol 2015;135:1264-7.

23 Bronstein AM, Golding JF, Gresty MA et al. The social impact of dizziness in London and Siena. J Neurol 2010;257:183-90.

$24 \mathrm{Na} \mathrm{PJ}$, Yaramala SR, Kim JA et al. The PHQ-9 Item 9 based screening for suicide risk: a validation study of the Patient Health Questionnaire (PHQ)-9 Item 9 with the Columbia Suicide Severity Rating Scale (C-SSRS). J Affect Disord 2018:232:34-40.

Address for correspondence: Mr David Herdman, Department of Health Psychology, King's College London, Guys Hospital, London SE1 9RT, UK.

Email: david.herdman@kcl.ac.uk

\section{Acute care toolkit 15: Managing acute care problems in pregnancy}

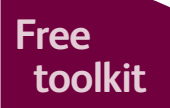

Pregnant women can present to any acute hospital service at any time during their pregnancy or the postpartum period, up to 12 months post-delivery. Women may present with acute medical problems that need to be managed differently because of pregnancy, or may present with obstetric syndromes.

This toolkit provides practical guidance on managing women with acute medical problems in pregnancy. It also advises healthcare staff to take the opportunity to ask about a woman's mental wellbeing when she comes to hospital for other reasons during or after pregnancy; women with a history of mental health problems are more likely to develop new symptoms during pregnancy and postpartum.

Essential reading for front-line NHS staff who may be unfamiliar with the normal physiology of pregnancy and/or diseases that present in pregnancy.

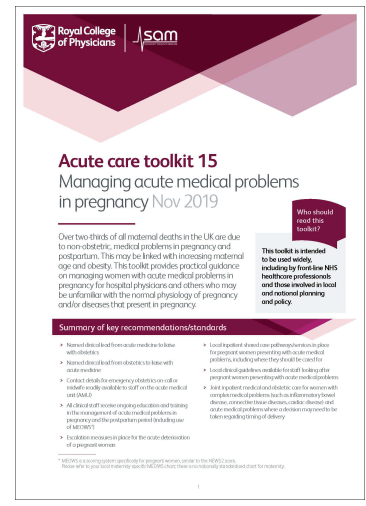

Download now at: www.rcplondon.ac.uk/act15 